\title{
Stanniocalcin 2 enhances mesenchymal stem cell survival by suppressing oxidative stress
}

\author{
Pyung-Hwan Kim ${ }^{1,2}$, Sang-Su Na ${ }^{2}$, Bomnaerin Lee ${ }^{2}$, Joo-Hyun $\mathrm{Kim}^{2}$ E Je-Yoel Cho ${ }^{2, *}$ \\ ${ }^{1}$ Department of Biomedical Laboratory Science, College of Medical Science, Konyang University, Daejeon 35365, Korea, ${ }^{2}$ Department of \\ Biochemistry, BK21 PLUS Program for Creative Veterinary Science Research, College of Veterinary Medicine, Seoul National University, \\ Seoul 08826, Korea
}

To overcome the disadvantages of stem cell-based cell therapy like low cell survival at the disease site, we used stanniocalcin 2 (STC2), a family of secreted glycoprotein hormones that function to inhibit apoptosis and oxidative damage and to induce proliferation. STC2 gene was transfected into two kinds of stem cells to prolong cell survival and protect the cells from the damage by oxidative stress. The stem cells expressing STC2 exhibited increased cell viability and improved cell survival as well as elevated expression of the pluripotency and self-renewal markers (Oct4 and Nanog) under sub-lethal oxidative conditions. Up-regulation of CDK2 and CDK4 and down-regulation of cell cycle inhibitors p16 and p21 were observed after the delivery of STC2. Furthermore, STC2 transduction activated pAKT and pERK 1/2 signal pathways. Taken together, the STC2 can be used to enhance cell survival and maintain long-term stemness in therapeutic use of stem cells. [BMB Reports 2015; 48(12): 702-707]

\section{INTRODUCTION}

With respect to cell-based therapy, there is no doubt that stem cells represent very special and excellent therapeutic tools due to their multipotent capacity to differentiate into various tissues, their self-renewal abilities, and their high degree of plasticity (1). Stem cells are also a smart delivery system to express or secret therapeutic factors in damaged organs as therapeutic agents and may represent a potential targeted gene delivery system. However, their clinical applications are limited despite their high therapeutic potential.

One of the major obstacles in the use of stem cells for cell

*Corresponding author. Tel: +82-2-880-1268; Fax: +82-2-886-1268; E-mail: jeycho@snu.ac.kr

http://dx.doi.org/10.5483/BMBRep.2015.48.12.158

Received 23 July 2015, Revised 13 August 2015, Accepted 25 September 2015

Keywords: Mesenchymal stem cells, Oxidative stress, Stanniocalcin2, Stem cell-based therapy therapy is the low survival rate in tissues and the limited expansion number. Stem cells are faced with dangerous challenges, such as oxidative stress, nutrient deprivation, host immune response, and oxygen deprivation when injected at the disease area, especially ischemic sites (2). Moreover, stem cells need to expand to obtain sufficient cell numbers (routinely 10-400 million mesenchymal stem cells per treatment) to achieve efficient therapeutic effects in vivo $(3,4)$. Among various factors responsible for the successful treatment of stem cell therapy is a reduction in the length of the cell expansion period in vitro and increased cell survival before the transplantation or injection. All primary cells, including stem cells or various origins, are limited in the number of cell divisions they can undergo under culture conditions $(5,6)$.

Recent studies indicate that the overexpression of anti-apoptotic and antioxidant proteins, such as the telomerase catalytic subunit or lipocalin 2, promotes stem cell resistance against ischemic stresses, thus increasing their viability and survival under harsh conditions $(2,7,8)$. Thus, increased stem cell therapeutic efficacy is required to maintain their long-term cell survival and to reduce the limitation of the time-dependent cell passage and cell expansion period in vivo.

Stanniocalcin 2 (STC2) is a member of the stanniocalcin family and is a peptide hormone regulating calcium and phosphate homeostasis $(9,10)$. The function of stanniocalcin 1 (STC1) is known to have various biological effects involving the inhibition of apoptosis and oxidative damage in various human cancers (11-13). For example, STC1 was reported to protect retinal ganglion cells by inhibiting apoptosis and oxidative damage (14), and STC1 secreted from MSCs protected bovine intestinal epithelial cells from oxidative damage (15).

However, whether STC family members are pro- or anti-apoptotic remains a controversial issue. Some reports indicate that STC1 is pro-apoptotic in chondrocytes during bone development (16). On the other hand, another report demonstrated anti-apoptotic function in heart and brain under hypoxia conditions (17). Although positive effects of STC1 are well-known in various cancers, the action and mechanism of STC2 in human cells, including stem cells, has yet to be fully understood. Furthermore, the relationship between STC2 and stem cells has not been reported thus far, and there are no 
studies concerning how STC affects stem cells.

Therefore, we investigated the biological function and mechanism of STC2 in adipose-derived stromal cells (ADSC), and umbilical cord blood-derived mesenchymal stem cells (UCBMSC) under oxidative damage to explore potential therapies to overcome the limitation of stem cell-based therapy.

\section{RESULTS}

\section{STC2 expression under oxidative stress}

After the decision of the sub-lethal concentration of $\mathrm{H}_{2} \mathrm{O}_{2}$ to stem cells (Supplementary Fig. 1), to access STC2 expression levels under conditions of the oxidative stress and to determine whether STC2 plasmid transfection into stem cells is expressed under the same conditions, RT-PCR was conducted using RNA extracted from cells treated with $\mathrm{NC}, \mathrm{H}_{2} \mathrm{O}_{2}$, pcDNA $+\mathrm{H}_{2} \mathrm{O}_{2}$, and pcDNASTC2 $+\mathrm{H}_{2} \mathrm{O}_{2}$. Under the normal condition (NC group), each stem cell expressed low levels of STC2. However, STC2 expression was significantly decreased in both cells in the presence of $\mathrm{H}_{2} \mathrm{O}_{2}$, as shown in inset graphs of Fig. 1 ( $P<0.05$ in ADSC). However, the cells transfected with the pcDNASSTC2 plasmid exhibited significantly high levels of STC2 expression compared with those transfected with pcDNA. These results demonstrate that the STC2 plasmid is well expressed in stem cells even under conditions of oxidative damage.

\section{Increased cell proliferation by STC2 overexpression}

We next tested the effect of STC2 on cell growth because the STC family is known to play an anti-apoptotic role in response to oxidative stress (9). Accordingly, we sought to determine
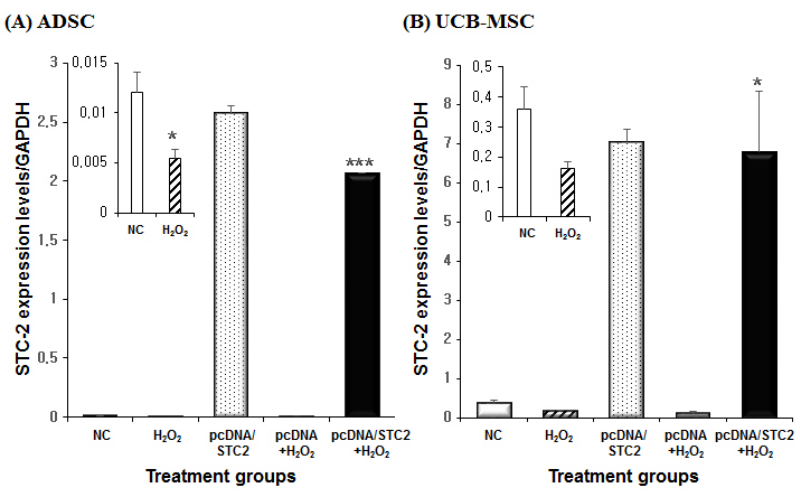

Fig. 1. Evaluation of STC2 expression levels in stem cells. Cells were transfected with pcDNA or pcDNA/STC2 plasmids. After 48 hrs, cells were treated with $100 \mathrm{mM} \mathrm{H}_{2} \mathrm{O}_{2}$ for $3 \mathrm{hrs}$, and then RNA was extracted and CDNA was synthesized, RT-PCR was conducted to detect STC2 expression in ADSC (A) and in UCB-MSC (B). Data represent the means and standard errors of triplicate experiments. ${ }^{*} \mathrm{P}<0.05$ for comparison of $\mathrm{H}_{2} \mathrm{O}_{2}$ against $\mathrm{NC}$ groups and $* * * \mathrm{P}<0.01$ versus pcDNA $+\mathrm{H}_{2} \mathrm{O}_{2}(\mathrm{~A})$, and $* \mathrm{P}<0.05$ versus $\mathrm{H}_{2} \mathrm{O}_{2}(\mathrm{~B})$. whether STC2 expression in ADSCs and UCB-MSCs can increase cell proliferation.

Fig. 2A depicts the cell morphologies after $\mathrm{H}_{2} \mathrm{O}_{2}$ treatment in STC2-expressing ADSCs. Cell death was induced in the cells treated with $\mathrm{H}_{2} \mathrm{O}_{2}$ compared with untreated cells in normal condition (NC) $37 \%$ or $78 \%$ cell viability in ADSCs or UCBMSCs, respectively, Fig. 2B for ADSCs and Supplementary Fig. 2A for UCB-MSCs). Similar rates of cell death were observed in the cells treated with the pcDNA vector in ADSCs. However, STC2-expressing cells exhibited a 1.8-fold increase in cell sur-

(A)
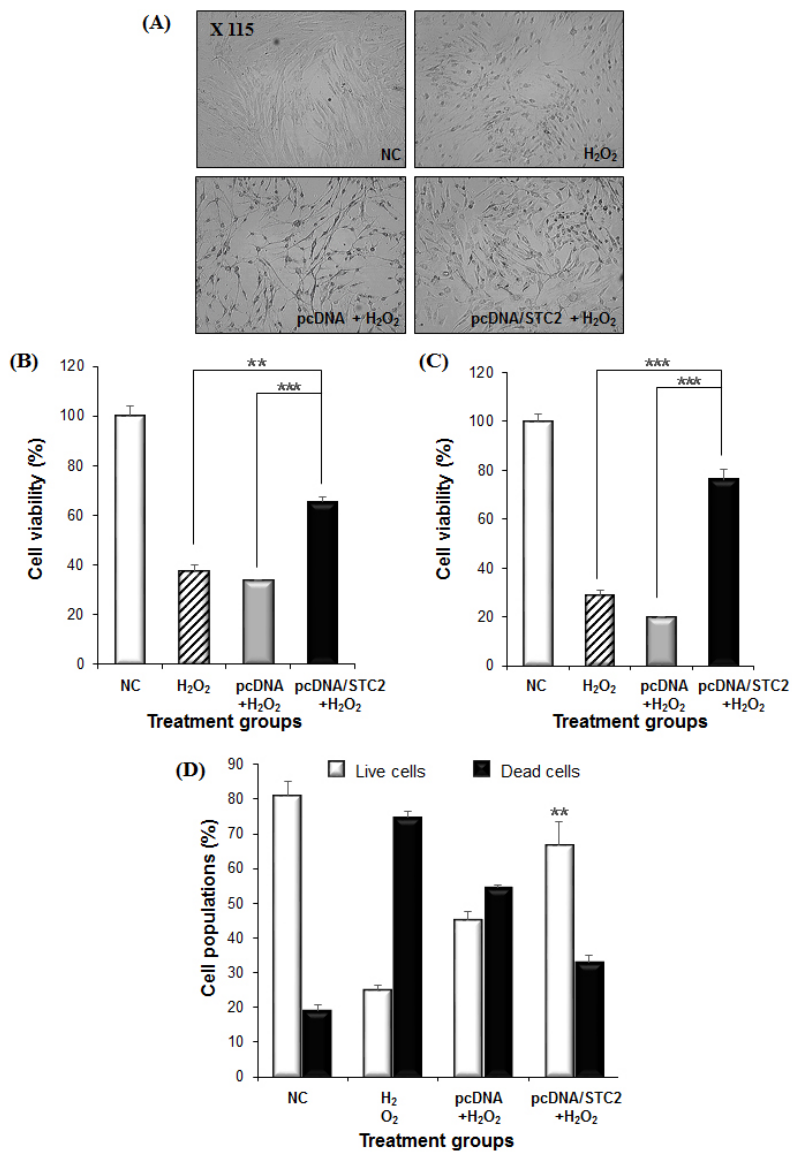

Fig. 2. Increased cell proliferative activity and enhancement of live/dead cell populations by STC2 expressed. At 48 hrs postSTC2 delivery, $\mathrm{H}_{2} \mathrm{O}_{2}$ was treated to cells for $3 \mathrm{hrs}$ and then cell morphology was observed (A) and MTT assay were performed (B). Another groups were assessed at 4 days post-media change of $\mathrm{H}_{2} \mathrm{O}_{2}$ treatment $(\mathrm{C})$. Increased cell proliferation and viability were observed in the cells transfected with STC2 plasmid. Data represent the means and standard errors of triplicate experiments. $* * \mathrm{P}<$ 0.02 and $* * * \mathrm{P}<0.01$ for comparison of pcDNASSTC2 $+\mathrm{H}_{2} \mathrm{O}_{2}$ groups against $\mathrm{H}_{2} \mathrm{O}_{2}$ or pcDNA $+\mathrm{H}_{2} \mathrm{O}_{2}$ groups, respectively. Also, cell viability of ADSCs was observed by Arthur image-based cytometer in cells stained with $\mathrm{Pl}$ solution after the treatment of STC2 and $\mathrm{H}_{2} \mathrm{O}_{2}$ (D). Dead cells were stained by $\mathrm{Pl}$ as red color. $* * \mathrm{P}<0.02$ versus $\mathrm{H}_{2} \mathrm{O}_{2}$ groups. 
vival in response to $\mathrm{H}_{2} \mathrm{O}_{2}$ stress. Four days after the treatment, increased cell death upon $\mathrm{H}_{2} \mathrm{O}_{2}$ stress was observed in the pcDNA-treated group compared with the results in Fig. 2B (Fig. 2C for ADSCs and Supplementary Fig. 2B for USC -MSCs). The pattern of results at this time was similar to that Fig. 2A, demonstrating that STC2 improves cell viability and proliferation upon $\mathrm{H}_{2} \mathrm{O}_{2}$ challenges. Taken together, these results indicate that STC2 can overcome oxidative stress-induced cellular damage.

\section{Live and dead cell populations}

Because STC can act as a factor to regulate cell survival, we evaluated cell viability after cellular damage. Cell viability was evaluated by counting the number of cells stained by $\mathrm{Pl}$, which is capable of discriminating live and dead cells. After staining, cells were analyzed by Arthur imaged-based cytometry. The quantification of live and dead cells treated with varying concentrations of $\mathrm{NC}, \mathrm{H}_{2} \mathrm{O}_{2}, \mathrm{pcDNA}+\mathrm{H}_{2} \mathrm{O}_{2}$, and pcDNA/STC2 $+\mathrm{H}_{2} \mathrm{O}_{2}$ is reported as the percentage of the cell population.

Fig. 2D shows the percent of cell stained by PI with respect to the total population as well as the percent of the total population that is both viable and expresses PI. The live and dead populations represented 81 and 19\% of the parental cells, respectively. Larger dead cell populations were observed in the cells treated with $\mathrm{H}_{2} \mathrm{O}_{2}(25 \%$ for live and $75 \%$ for dead cells). However, increased live cell and decreased dead cell populations were observed in the cells treated with pcDNA/STC2 $+\mathrm{H}_{2} \mathrm{O}_{2}(67$ and $33 \%)$ compared with those of the cells treated with pcDNA $+\mathrm{H}_{2} \mathrm{O}_{2}(45$ and $55 \%)$. These data demonstrate that STC2 expression in ADSCs elicits enhanced cell survival compared with empty vector transfected cells in response to oxidative stress.

\section{Biological potential of ADSCs expressing STC2}

The increased cell proliferation induced by STC2 may also be caused by the up-regulation of cell cycle-related proteins. To compare the expression levels of cell cycle regulators, we measured the levels of cyclin-dependent kinases (CDKs) and their inhibitor proteins $\mathrm{p} 16$ and p21 by RT-PCR in ADSCs treated with $\mathrm{NC}, \mathrm{H}_{2} \mathrm{O}_{2}$, and pcDNA/STC2 $+\mathrm{H}_{2} \mathrm{O}_{2}$.

As shown in Fig. 3A, CDK2 or CDK4 expression was increased in the cells treated with pcDNA/STC2 $+\mathrm{H}_{2} \mathrm{O}_{2}$ under oxidative conditions. CDK2 and CDK4 expression were about 5.8- and 3.2-fold higher, respectively, in the cells transfected with pcDNA/STC2 than in the cells treated with $\mathrm{H}_{2} \mathrm{O}_{2}$ alone. These results were also confirmed in UCB-MSCs (Supplementary Fig. 3). In the case of the CDK inhibitor proteins, CDK inhibitor $2 \mathrm{~A}$ and $1 \mathrm{~A}$ (also known as $\mathrm{p} 16$ and $\mathrm{p} 21$, respectively), the cells transfected with pcDNA/STC2 $+\mathrm{H}_{2} \mathrm{O}_{2}$ exhibited approximately 2.6-and 1.1-fold lower p16 and p21 expression than those of parental cells when treated with $\mathrm{H}_{2} \mathrm{O}_{2}$ (Fig. 3B). These results demonstrate that elevated expression of CDK2 and CDK4 and decreased expression p16 is at least responsible for the increased cell viability induced by STC2.
Because cell viability against oxidative stress-induced cellular damage was increased by STC2, we next assessed whether the multipotency of stem cells is maintained by STC2 expression in ADSCs. Our results revealed that pcDNA/STC2+ $\mathrm{H}_{2} \mathrm{O}_{2}$ exhibited higher expression of stemness factors (Nanog and Oct4) than the $\mathrm{H}_{2} \mathrm{O}_{2}$ groups alone $(\mathrm{P}<0.02$ for Nanog and $\mathrm{P}<0.05$ for Oct4) (Fig. 3C). Intriguingly, Nanog and Oct4 expression in cells treated with STC2 were more highly induced under oxidative conditions than under normoxic conditions ( $\mathrm{P}<0.02$ for Oct4). These data imply that STC2 transfection leads to maintained biological potency in stem cells
(A)

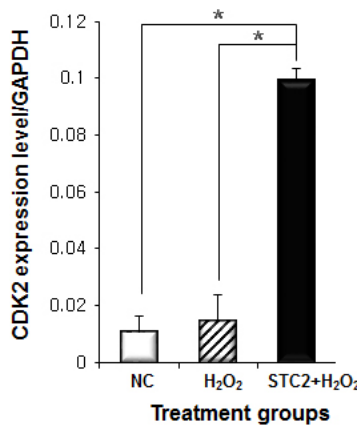

(B)

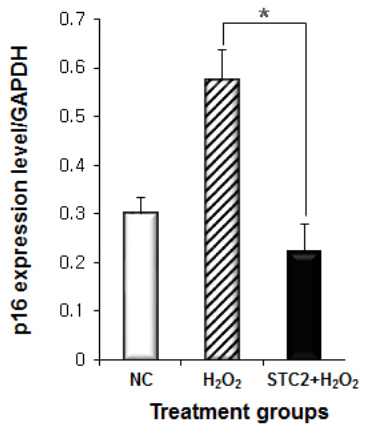

(C)

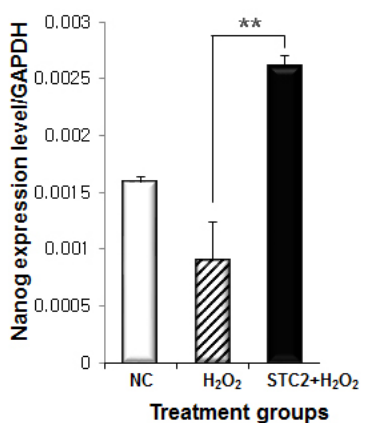

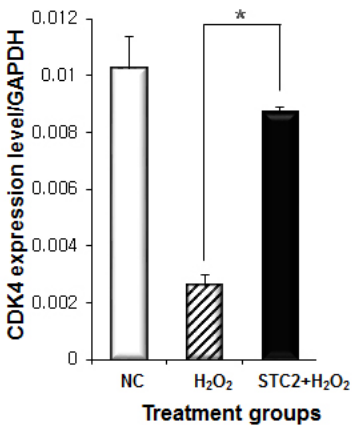
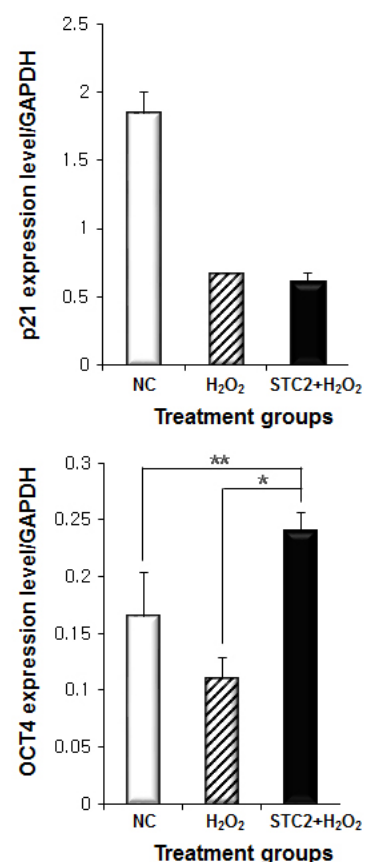

Fig. 3. Biological activity of STC2 expressed in ADSC. The expression levels of cell cycle regulator proteins, CDK2 and $4(\mathrm{~A})$, inhibitor proteins, p16 and p21 (B), and stemness factors, Nanog and Oct4 (C). ADSCs were treated with $\mathrm{NC}, \mathrm{H}_{2} \mathrm{O}_{2}$, and pcDNASTC2+ $\mathrm{H}_{2} \mathrm{O}_{2}$, and then got oxidative damage for 3 hrs. After RNA extraction and cDNA synthesis, the expression of each factors were measured by RT-PCR. Data represent means \pm SE. ${ }^{* P}<0.05$ and $* * P<0.02$ for remarked groups. 


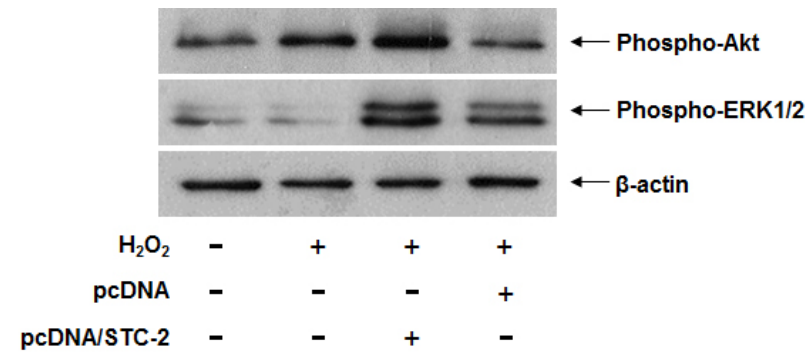

Fig. 4. Investigation of the mechanism about improved positive effects in stem cells. Cellular signal pathway was examined by western blot. Phosphotylated Akt and ERK1/2 levels up-regulated in STC2 expressed ADSC under oxidative stress. pcDNA $+\mathrm{H}_{2} \mathrm{O}_{2}$ groups were used as a negative control and $\beta$-actin was used as internal control.

subjected to cellular damage caused by oxidative stress.

\section{STC2 signaling pathway confers improved stem cell survival} under oxidative stress

To explore the mechanism by which STC2 induces improved stem cell viability against oxidative stress, the expression levels of phospho-protein kinase B (pAkt) and phospho-extracellular signal-regulated kinase $1 / 2$ (pERK $1 / 2$ ), which play major roles in cell survival and proliferation, were examined by western blot analysis.

As shown in Fig. 4, reduction of pAkt and pERK 1/2 was observed in the cells treated with $\mathrm{H}_{2} \mathrm{O}_{2}$. In contrast, STC2 transfection increased the protein levels relative to the pcDNA groups despite treatment with $\mathrm{H}_{2} \mathrm{O}_{2}$. These data indicate that the activation of Akt and ERK 1/2 by STC2 might up-regulate cell cycle regulators and stemness factors and thus increase cell survival upon oxidative stress.

\section{DISCUSSION}

Stem cell-based cell therapies derived from various sources have been applied in over 250 ongoing clinical trials (18). Although the benefits of stem cell therapy have been reported for a variety of diseases, some limitations exist that complicate successful clinical trials using stem cells. The major hurdles include how to overcome the heterogeneity of stem cell populations and cell senescence as well as cryopreservation and in vitro expansion for clinical application.

Death during the cell expansion period and death induced by environmental conditions, such as hypoxia or oxidative damage, represent additional challenges that may be overcome by taking advantage of the overexpression of anti-apoptotic and antioxidant proteins to improve cell survival. Another approach is to pursue large-scale expansion in bioreactors (19), induce enhanced stem cell trophic functions $(20,21)$ or encapsulate stem cells with biomaterials such as microcapsules (22-24). Increasing stem cell survival through various strategies will promote long-term therapeutic efficacy.

Therefore, we focused on a protein up-regulated in cancer, stanniocalcin 2 (STC2). STC2 is highly expressed in hepatocellular carcinoma (25) and breast cancer (26) as well as in human tissues such as skeletal muscle, heart, and pancreas (27). STC2 also promotes anti-apoptotic and pro-proliferative action in cancer. We applied the protein to provide stem cells with the survival properties of cancer.

In the present study, we administered $\mathrm{H}_{2} \mathrm{O}_{2}$ to two types of stem cells, ADSCs and UCB-MSCs to mimic oxidative stress-induced cellular damage in ischemic sites in vivo. In the case of cancer, the expression of stanniocalcin family members is induced under oxidative condition. However, the levels of STC2 in stem cells were down-regulated under the same conditions (Fig. 1). Consequently, the cell viability and proliferation rate were reduced, leading to cell death. Cell damage was accelerated by oxidative stress. However, STC2 can promote recovery against cell damage under oxidative conditions. When STC2 is expressed in stem cells, cell viability and proliferation were increased after exposure to $\mathrm{H}_{2} \mathrm{O}_{2}$ at early and late time points, as shown in Fig. 2. These patterns were observed in another stem cell, UCB-MSC. The results indicate that STC2 exhibits pleiotropic effects by increasing the resistance of stem cells to microenvironmental damage. These facts were confirmed by measuring the live and dead cell populations in Fig. 2D. The major causes of the low therapeutic effects of stem cells in vivo are associated with immune rejection, anoikis, and oxidative damage-mediating apoptosis (3, 28-30). In our study, the live cell population was significantly reduced following treatment with $\mathrm{H}_{2} \mathrm{O}_{2}$ but was largely unaffected after treatment with STC2, suggesting that STC2 can promote cell survival under stressful circumstances.

The improved cell viability and proliferation may also be caused by increased cell cycle proteins. Cyclin-dependent kinases (CDKs) regulate the cell cycle in complex with their catalytic subunits (31). The activities of CDK2 and 4 are known to be restricted to the G1-S phase of the cell cycle and are essential for the G1/S transition $(32,33)$. Additionally, CDK2 or 4 are controlled by the CDK inhibitors p21Cip1 (CDKN1A), p27Kip1 (CDKN1B), and p16 ${ }^{\text {INK4a }}(34,35)$.

Based on the facts described above, we have evaluated the expression levels of cell cycle-related proteins because CDk2 and 4 as well as p16 and 21 are responsible for G1/S progression with cyclins. The levels of cell cycle proteins were up-regulated by STC2 despite $\mathrm{H}_{2} \mathrm{O}_{2}$ treatment, but the expression of the CDK inhibitor proteins, p16 and p21, were down-regulated (Fig. 3A and 3B). Hydrogen oxidase-treated cells were arrested in $\mathrm{G} 1$ or at G1/S phase compared with the non-treated cells. In addition to their function as CDK inhibitor proteins, p16 and p21 also exhibit roles in senescence and are known to be senescence markers. Cell senescence progressively increases with passage number, leading to cell death. Fig. 3B demonstrates low expression levels of p16 and p21 in STC2-expressing cells after $\mathrm{H}_{2} \mathrm{O}_{2}$ treatment, which may 
indicate greater survival advantages in hazardous circumstance. Taken together, these results indicate that the expression of STC2 induces the up-regulation of stem cell cycle proteins and protects against oxidative stress, leading to increased proliferation.

A major reason for the clinical application of stem cell is their multi-pluripotency and self-renewal capacity (36). The long-term expression of pluripotency markers is essential for the improvement of the therapeutic efficacy $(37,38)$.

Thus, to verify these facts, we assessed whether Nanog and Oct4, representative pluripotency markers, were maintained by STC2 expression in stem cells under the conditions of cellular damage. In Fig. 3C, multipotency markers were significantly induced compared with those of the $\mathrm{H}_{2} \mathrm{O}_{2}$-treated group despite the harmful environment, indicating that prolonged marker expression may allow STC2-expressing stem cells to improve therapeutic efficacy and regenerative capacity in hazardous environments.

We accordingly evaluated the potential molecular mechanism by which these positive effects were observed upon STC2 expression. Fig. 4 demonstrates that the pERK1/2 and pAkt signal increased after STC2 expression despite $\mathrm{H}_{2} \mathrm{O}_{2}$ treatment compared with the control groups. Consequently, the enhanced cell survival and proliferation in response to STC2 expression may be caused by elevated activity of the ERK and Akt signaling pathways. Additionally, ERK expression levels are responsible for promoting the $\mathrm{G} 1 / \mathrm{S}$ phase of the cell cycle. The expressed STC2 in ADSCs promoted cell cycle progression downstream of increased regulatory proteins (e.g., CDKs) under oxidative stress through ERK activation. Taken together, STC2 promoted enhanced up-regulation of the ERK and Akt pathway accompanied by increased cell survival and proliferative activity, supporting the potential application of STC2 in stem cell therapies.

To improve stem cell-based therapy, stem cells must overcome poor cell survival and the loss of multi-pluripotency during cell expansion before clinical trials as well as ischemic conditions at disease sites to increase therapeutic potency. In the present study, we used STC2 to improve the therapeutic function of stem cells by maintaining stemness factors and increasing cell survival and proliferative activity under oxidative stress-induced cellular damage. This research is the first report focused on the effects of STC2 on stem cells. Increased cell proliferation and survival were observed in ADSCs and UCB-MSCs expressing STC2 after exposure to $\mathrm{H}_{2} \mathrm{O}_{2}$. When STC2 was delivered into the cells, up-regulation of cell cycle regulator proteins and down-regulation of cell cycle inhibitors was induced relative to the treatment of the cells with $\mathrm{H}_{2} \mathrm{O}_{2}$ or control vector. Moreover, high expression of pluripotency markers was observed and maintained in the cells treated with STC2 despite oxidative conditions. High activation of ERK and Akt accompanied the improved positive effects on stem cells. Together, our results indicate that the expression of a paradoxical gene in stem cells may be a promising strategy to im- prove multipotent capacity and to overcome the in vivo limitations for a variety of clinical applications, indicating that STC2 may induce the long-term therapeutic efficacy of stem cells.

\section{MATERIALS AND METHODS}

\section{Cell lines and STC2 plasmid construct}

Human adipose-derived mesenchymal stem cells (ADSC) and human mesenchymal stem cells isolated from umbilical cord blood (UCB-MSC) were kindly provided from EHL Biotechnologie Institute and Dr. Kyung Sun Kang at Seoul National University in Seoul, Republic of Korea, respectively. The human UCB-MSC isolation procedure was approved by the Borame Hospital Institutional Review Board and Seoul National University (IRB No. 0603/001-002-07C1). ADSC, UCB-MSC cultured in Dulbecco's Modified Eagle's Medium (DMEM; Gibco-BRL, Grand Island, NY) with $20 \%$ fetal bovine serum (FBS; Gibco-BRL), 10\% for $\mathrm{H} 460$ and penicillin/streptomycin (Gibco-BRL). They were maintained at $37^{\circ} \mathrm{C}$ and $5 \% \mathrm{CO}_{2}$.

To create stanniocalcin2 (STC2) expressing pDNA, STC2 cDNA was amplified by PCR from human lung cancer H460 (purchased from ATCC). The forward and reverse primers for STC2 were 5'-GGATCCTACACACGGCTCGCCGAC-3' and 5'CTCGAGTCACCTCCGGATATCAGA-3', respectively. The BamHI and $X$ hol sites were introduced into the forward and reverse primers, respectively (the enzyme sites are underlined). The amplified STC2 was inserted into pGEM ${ }^{\circledR}$-T Vector (Promega, Madison, WI), and then was digested with $\mathrm{BamHI}$ and Xhol and then purified by electrophoretic elution from a $1 \%$ agarose gel. pcDNA/STC2 was constructed by insertion of the STC2 fragment into the site of pcDNA3.1 (Life technologies, Grand Island). Each cloning steps were confirmed by restriction enzyme digestion (data not shown). Most of contents of Materials and Methods were described in supplementary data.

\section{ACKNOWLEDGEMENTS}

This research was supported by grants from the National Research Foundation (NRF) funded by the Ministry of Science, ICT \& Future Planning (2012M3A9C6049716) and Education (2013R1A1A2062949).

\section{REFERENCES}

1. Mimeault M, Hauke R and Batra SK (2007) Stem cells: a revolution in therapeutics-recent advances in stem cell biology and their therapeutic applications in regenerative medicine and cancer therapies. Clin Pharmacol Ther 82, 252-264

2. Estrada JC, Torres Y, Benguria A et al (2013) Human mesenchymal stem cell-replicative senescence and oxidative stress are closely linked to aneuploidy. Cell Death Dis 4, e691

3. Sart S, Ma T and Li Y (2014) Preconditioning stem cells for in 
vivo delivery. Biores Open Access 3, 137-149

4. Salem HK and Thiemermann C (2010) Mesenchymal stromal cells: current understanding and clinical status. Stem Cells 28, 585-596

5. Sotiropoulou PA, Perez SA, Salagianni M, Baxevanis $C N$ and Papamichail M (2006) Characterization of the optimal culture conditions for clinical scale production of human mesenchymal stem cells. Stem Cells 24, 462-471

6. Bonab MM, Alimoghaddam K, Talebian F, Ghaffari SH, Ghavamzadeh A and Nikbin B (2006) Aging of mesenchymal stem cell in vitro. BMC Cell Biol 7, 14

7. Bahmani B, Roudkenar MH, Halabian R, Jahanian-Najafabadi A, Amiri $F$ and Jalili MA (2014) Lipocalin 2 decreases senescence of bone marrow-derived mesenchymal stem cells under sub-lethal doses of oxidative stress. Cell Stress Chaperones $19,685-693$

8. Sakata H, Niizuma K, Wakai T, Narasimhan P, Maier CM and Chan $\mathrm{PH}$ (2012) Neural stem cells genetically modified to overexpress cu/zn-superoxide dismutase enhance amelioration of ischemic stroke in mice. Stroke 43, 2423-2429

9. Yeung BH, Law AY and Wong CK (2012) Evolution and roles of stanniocalcin. Mol Cell Endocrinol 349, 272-280

10. Wagner GF and Dimattia GE (2006) The stanniocalcin family of proteins. J Exp Zool A Comp Exp Biol 305, 769-780

11. Chang AC, Jellinek DA and Reddel RR (2003) Mammalian stanniocalcins and cancer. Endocr Relat Cancer 10, 359- 373

12. Tamura S, Oshima T, Yoshihara K et al (2011) Clinical significance of STC1 gene expression in patients with colorectal cancer. Anticancer Res 31, 325-329

13. Fujiwara $Y$, Sugita $Y$, Nakamori $S$ et al (2000) Assessment of Stanniocalcin-1 mRNA as a molecular marker for micrometastases of various human cancers. Int J Oncol 16, 799-804

14. Kim SJ, KoJH, Yun JH et al (2013) Stanniocalcin-1 protects retinal ganglion cells by inhibiting apoptosis and oxidative damage. PLoS One 8, e63749

15. Wu LM, Hui L, Guo R et al (2014) Stanniocalcin-1 protects bovine intestinal epithelial cells from oxidative stress-induced damage. J Vet Sci 15, 475-483

16. Wu S, Yoshiko Y and De Luca F (2006) Stanniocalcin 1 acts as a paracrine regulator of growth plate chondrogenesis. J Biol Chem 281, 5120-5127

17. Westberg JA, Serlachius $M$, Lankila P, Penkowa M, Hidalgo J and Andersson LC (2007) Hypoxic preconditioning induces neuroprotective stanniocalcin-1 in brain via IL-6 signaling. Stroke 38, 1025-1030

18. Levy O, Zhao W, Mortensen LJ et al (2013) mRNA-engineered mesenchymal stem cells for targeted delivery of interleukin-10 to sites of inflammation. Blood 122, e23-32

19. Baghbaderani BA, Mukhida K, Hong M, Mendez I and Behie LA (2011) A review of bioreactor protocols for human neural precursor cell expansion in preparation for clinical trials. Curr Stem Cell Res Ther 6, 229-254

20. Wang K, Zhang T, Dong Q, Nice EC, Huang C and Wei Y (2013) Redox homeostasis: the linchpin in stem cell self-renewal and differentiation. Cell Death Dis 4, e537

21. Das B, Bayat-Mokhtari R, Tsui M et al (2012) HIF-2alpha suppresses p53 to enhance the stemness and regenerative potential of human embryonic stem cells. Stem Cells 30, 1685-1695

22. Liu TC, Ismail S, Brennan O, Hastings C and Duffy GP (2013)
Encapsulation of cardiac stem cells in superoxide dismutase-loaded alginate prevents doxorubicin-mediated toxicity. J Tissue Eng Regen Med 7, 302-311

23. Zhong J, Chan A, Morad L, Kornblum HI, Fan G and Carmichael ST (2010) Hydrogel matrix to support stem cell survival after brain transplantation in stroke. Neurorehabil Neural Repair 24, 636-644

24. Kim PH, Yim HG, Choi YJ et al (2014) Injectable multifunctional microgel encapsulating outgrowth endothelial cells and growth factors for enhanced neovascularization. J Control Release 187, 1-13

25. Okabe H, Satoh S, Kato T et al (2001) Genome-wide analysis of gene expression in human hepatocellular carcinomas using cDNA microarray: identification of genes involved in viral carcinogenesis and tumor progression. Cancer Res 61, 2129-2137

26. Bouras T, Southey MC, Chang AC et al (2002) Stanniocalcin 2 is an estrogen-responsive gene coexpressed with the estrogen receptor in human breast cancer. Cancer Res 62, 1289-1295

27. Wang H, Wu K, Sun Y et al (2012) STC2 is upregulated in hepatocellular carcinoma and promotes cell proliferation and migration in vitro. BMB Rep 45, 629-634

28. de Almeida PE, Ransohoff JD, Nahid A and Wu JC (2013) Immunogenicity of pluripotent stem cells and their derivatives. Circ Res 112, 549-561

29. Le Blanc K (2006) Mesenchymal stromal cells: Tissue repair and immune modulation. Cytotherapy 8, 559-561

30. Hsiao ST, Dilley RJ, Dusting GJ and Lim SY (2014) Ischemic preconditioning for cell-based therapy and tissue engineering. Pharmacol Ther 142, 141-153

31. Satyanarayana A and Kaldis P (2009) Mammalian cell-cycle regulation: several Cdks, numerous cyclins and diverse compensatory mechanisms. Oncogene 28, 2925-2939

32. Hydbring P and Larsson LG (2010) Cdk2: a key regulator of the senescence control function of Myc. Aging (Albany NY) 2, 244-250

33. Gogolin S, Ehemann V, Becker G et al (2013) CDK4 inhibition restores $\mathrm{G}(1)$-S arrest in MYCN-amplified neuroblastoma cells in the context of doxorubicin-induced DNA damage. Cell Cycle 12, 1091-1104

34. Romanov VS, Pospelov VA and Pospelova TV (2012) Cyclin-dependent kinase inhibitor p21(Waf1): contemporary view on its role in senescence and oncogenesis. Biochemistry (Mosc) 77, 575-584

35. Sheppard KE and McArthur GA (2013) The cell-cycle regulator CDK4: an emerging therapeutic target in melanoma. Clin Cancer Res 19, 5320-5328

36. Tsai CC, Su PF, Huang YF, Yew TL and Hung SC (2012) Oct4 and Nanog directly regulate Dnmt1 to maintain self-renewal and undifferentiated state in mesenchymal stem cells. Mol Cell 47, 169-182

37. Baer PC, Griesche N, Luttmann W, Schubert R, Luttmann A and Geiger H (2010) Human adipose-derived mesenchymal stem cells in vitro: evaluation of an optimal expansion medium preserving stemness. Cytotherapy 12, 96-106

38. Go MJ, Takenaka C and Ohgushi H (2008) Forced expression of Sox2 or Nanog in human bone marrow derived mesenchymal stem cells maintains their expansion and differentiation capabilities. Exp Cell Res 314, 1147-1154 